\title{
Nicht-kleinzelliges Lungenkarzinom: Neues aus der Immuntherapie
}

\author{
Niels Reinmuth, David F. Heigener, Martin Reck
}

Wie alle Tumore tarnt sich auch das nicht-kleinzellige Lungenkarzinom gegenüber dem Immunsystem. Ein Ansatz in der Therapie ist, dass Antikörper diese Tarnung aufheben und die körpereigene Abwehr unterstützen. Erfahren Sie, welche immunmodulatorischen Therapieansätze derzeit in klinischen Studien getestet werden und wo vielversprechendes Potenzial liegt.

Immunreaktion | Die Bedeutung des Immunsystems in der Karzinogenese ist bislang nur in Ansätzen entschlüsselt. Das Mikromilieu des Tumors besteht aus

- Immunzellen,

- Stromazellen und

- Tumorzellen.

Dort entfalten insbesondere Lymphozyten und Makrophagen über Zytokin- und Chemokinkaskaden komplexe und teils widersprüchlich scheinende Wirkungen. Folgende Szenarien sind bei der Reaktion des Immunsystems gegen den Tumor möglich:

- Eliminierung von Tumorzellen

- Förderung der Tumorentstehung durch immunologische Faktoren [1, 2]. Dies gilt v.a. für etablierte solide Tumoren, da dort die Tumorsurveillance nur begrenzt wirksam ist.

Auch beim nicht-kleinzelligen Lungenkarzinom (NSCLC) finden sich häufig dichte Infiltrate der verschiedenen T-Zell-Populationen. Die Korrelation mit der Prognose wird dabei kontrovers diskutiert [3]. In einer Metaanalyse korreliert die Zahl intratumoraler T-Zellen jedoch positiv mit dem Überleben [4].

Immuntherapie I Da das Immunsystem so komplex ist, galt eine immunologische Tumortherapie lange als kaum möglich. Eine Immuntherapie wirkt durch eine Interaktion zwischen Antigen und Immunsystem. Ziel dabei ist, das Tumorwachstum durch körpereigene Abwehrmechanismen zu beeinflussen. In den vergangenen Jahrzehnten wurde - überwiegend präklinisch - intensiv geforscht. Ein immer besseres Verständnis der komplexen immunregulatorischen Mechanismen war die Folge. Derzeit werden verschiedene immun-modulierende Therapieansätze klinisch entwickelt.

Die entwickelten immun-modulierenden Therapieansätze können Antigen-unspezifisch und/ oder Antigen-spezifisch sein.

\section{Impfung}

Ziel | Die Antigen-spezifische Impfstrategie soll

- die Bildung Tumorantigen-spezifischer Antikörper sowie

- zytotoxischer CD4- und CD8-T-Zellen auslösen,

- das Immunsystem des Patienten gegen spezifische Tumorantigene sensibilisieren und

- Mechanismen einer Tumor-induzierten Toleranz umgehen.

Voraussetzung | Eine wichtige Voraussetzung hierfür ist eine effektive Präsentation eines Tumorantigens durch Antigen-präsentierende Zellen (dendritische Zellen, Makrophagen, BLymphozyten). Somit werden Tumor-spezifische T-Zellen stimuliert [5].

Das auslösende Antigen sollte idealerweise

- tumorspezifisch sein

- sich von gesunden Zellen unterscheiden

- immunogen wirken

- auch bei fortgeschrittener Erkrankung auf den Tumorzellen nachweisbar bleiben [6, 7]

L-BLP25 I Die liposomale BLP25-Vakzine (LBLP25, Tecemotide) richtet sich gegen das muzinöse Glykoprotein-1 (MUC1). Dieses wird normalerweise auf der Oberfläche Muzin-sezernierender Epithelzellen exprimiert. Bei Tumorzellen wird das Protein überexprimiert oder aberrant glykosyliert [8]. Eine Phase-II-Studie lieferte initial vielversprechende Ergebnisse. Diese konnten in einer Phase-III-Studie (START) an 1513 Patienten mit inoperablem NSCLC im Stadium III jedoch nicht bestätigt werden: L-BLP25 brachte keinen signifikanten Überlebensvorteil (Median 25,6 vs. 22,3 Monate; Hazard Ratio [HR] 0,88; 95\%-Konfidenzintervall [KI] 0,75-1,03; p=0,123) [9]. Die Vakzinierung wurde bis auf leichte Grippe-ähnliche Symptome und lokale Entzündungsreaktionen gut toleriert.

Überlebensvorteil durch L-BLP25-Vakzin? | Eine explorative Subgruppenanalyse zeigte, dass Patienten nach simultaner Radiochemotherapie durch 
Abb. 1 Checkpoint CTLA 4 (im Lymphknoten). Die Präsentation eines Antigens über den Major Histocompability complex (MHC) an den T-Zell Rezeptor (TZR) führt mithilfe der Co-Stimulation von B7 an CD28 zur T-Zell-Aktivierung. Bindet $B 7$ jedoch an CTLA 4 wird die Aktivierung verhindert. eine L-BLP25-Vakzinierung profitieren könnten: medianes Überleben 30,8 vs. 20,6 Monaten; HR 0,78; 95\%-KI 0,64-0,95; p=0,016. Nach einer Pressemitteilung erbrachte aber eine - derzeit noch unpublizierte - randomisierte japanische PhaseI/ II-Studie für das gleiche Patientenkollektiv nach simultaner oder sequentieller Chemoradiotherapie ebenfalls keinen Vorteil für das Gesamtüberleben und das progressionsfreie Überleben.

In der Folge wurde die klinische Entwicklung der L-BPL25-Impfung bei NSCLC weltweit gestoppt.

MAGE-A3 | Ein anderer Vakzinierungsansatz richtet sich gegen das Protein „melanoma-associated antigen 3“" (MAGE-A3). Es wird nahezu ausschließlich von Tumorzellen exprimiert und kann bei $24-50 \%$ der NSCLC-Fälle detektiert werden [6]. Eine kürzlich vorgestellte Phase-III-Studie (MAGRIT) untersuchte eine 27-monatige MAGE-A3-Vakzinierung bei 2272 Patienten mit komplett reseziertem NSCLC im Stadium IB-IIIA. Alle Teilnehmer exprimierten das Antigen [10]. Im Vergleich zu Placebo konnte die Impfung das krankheitsfreie Überleben in der Gesamtpopulation nicht verlängern (Median 60,5 vs. 57,9 Monate; HR 1,024; 95\%-KI 0,891-1,177; $\mathrm{p}=0,74$ ). Gleiches galt für die Untergruppe von Patienten ohne adjuvante Chemotherapie (Median 58,0 vs. 56,9 Monate; HR 0,970; $\mathrm{p}=0,7572$ ).

Allogene Tumorvakzine | Belagenpumatucel-L ist eine allogene Tumorvakzine, die aus vier verschiedenen NSCLC-Zelllinien hergestellt wird. Um die Immunogenität zu verstärken, werden die Zellen mit einem Anti-transforming growth factor$\beta 2$-Plasmid transfiziert. Eine Phase-III-Studie (STOP) prüfte eine monatliche Erhaltungstherapie nach Erstlinien-Chemo(strahlen)therapie bei NSCLC Stadium III oder IV ( $n=532)$ [11]. Das mediane Gesamtüberleben war im Vergleich zu Placebo nicht signifikant verlängert ( 20,3 vs. 17,8 Monate; HR 0,94; $p=0,594)$. An einer Subgruppenanalyse nahmen 305 Patienten im NSCLC-Stadium IIIB/IV teil, die innerhalb von 12 Wochen nach Chemotherapie randomisiert wurden. Hier war das Gesamtüberleben mit die Impfung verlängert (Median 20,7 vs. 13,4 Monate; HR 0,75; $\mathrm{p}=0,083)$.

Weitere Impfstrategien | Verschiedene weitere Ansätze befinden sich in klinischer Testung [12].

Dazu gehören z. B.

- Impfungen gegen Ganglioside,

- Impfungen gegen den epidermalen Wachstumsfaktor-Rezeptor (EGFR) oder

- alternative Vakzine gegen oben beschriebene Antigene (z.B. TG4010).

Mögliche Probleme | Möglicherweise könnte eine T-Zell-Aktivierung auch in der Anwesenheit von potenten Antigenen weniger stark ausgeprägt sein - insbesondere nach wiederholter Vakzinierung [13]. Weitere prädiktive Marker könnten notwendig werden, um geeignete Patientenkollektive zu definieren.

Als Schlüssel zu diesem wichtigen Schritt in der Immunantwort sind sogenannte „CheckpointInhibitoren“ denkbar.

\section{Checkpoint-Inhibitoren}

Prinzip von Checkpoint-Inhibitoren I Bei der Regulation des Immunsystems gibt es verschiedene Elemente, die die Immunantwort hemmen oder co-stimulieren, sogenannte „Checkpoints“. Mit der gezielten Inhibition dieser Checkpoint-Signale soll eine gesteigerte, zytotoxische T-Zell-Antwort erreicht werden.

Antikörper gegen CTLA-4 und PD-1 | Der Antikörper Ipilimumab richtet sich gegen das „cytotoxic T-lymphocyte antigen-4“ (CTLA-4). Er ist bereits beim Melanom zugelassen und befindet sich zur Zeit in der fortgeschrittenen klinischen Prüfung für den Einsatz bei NSCLC. Antikörper gegen das „programmed cell death protein“ (PD-1) und seine Liganden zeigen ebenfalls erste vielversprechende Ergebnisse.

Die Rolle von CTLA-4 I Das Protein CTLA-4 wird auf T-Zellen exprimiert. Es wird hochreguliert, wenn Antigen-präsentierende Zellen den T-ZellRezeptorkomplex aktivieren. In der Folge wird die Immunantwort abgeschwächt [14] ( Abb. 1).

Chemotherapie mit/ohne Ipilimumab I In einer randomisierten Phase-II-Studie wurden Patienten mit nicht-vorbehandeltem NSCLC drei Therapie-Armen zugeordnet:

- Carboplatin, Paclitaxel und Placebo

- Carboplatin, Paclitaxel und Ipilimumab gleichzeitig/,simultan“ (Zyklus 1 bis 4)

- Carboplatin, Paclitaxel und Ipilimumab verzögert/ „phasisch“ (Zyklus 3 bis 6) 
Nach 6 Zyklen wurden Ipilimumab oder Placebo alle drei Monate als Erhaltung gegeben.

Verzögerte Kombinationstherapie | Hinsichtlich des immunabhängigen progressionsfreien Überlebens (irPFS) war die Ipilimumab-ChemotherapieKombination der alleinigen Chemotherapie nur dann signifikant überlegen, wenn sie verzögert verabreicht wurde ( $\bullet$ Tab. 1 ). Auch das Gesamtüberleben war in der phasischen Gruppe numerisch besser als in der simultanen Therapiegruppe (beide Unterschiede nicht signifikant). Zudem waren in der phasischen Gruppe die Therapieeffekte bei Plattenepithelkarzinomen stärker ausgeprägt (irPFS, HR $=0,55$ ) als bei Nicht-Plattenepithelkarzinomen $(H R=0,82)$. Die Ergebnisse werden derzeit in einer Phase III-Studie überprüft (phasische Applikation von Ipilimumab+Carboplatin + Paclitaxel vs. alleinige Chemotherapie bei squamösem NSCLC).

Die Rolle von PD1 und PD-L1 I PD1 ist ebenfalls ein Rezeptor auf der Oberfläche der T-Zelle. Er supprimiert die Immunantwort und greift in verschiedene Regelmechanismen der Immunantwort ein [16] ( Abb. 2). Fibroblasten aus dem Tumorstroma und auch Tumorzellen selbst können Liganden (PD-L1 und PD-L2) sezernieren und somit die Immunantwort auf den Tumor verändern. Verschiedene Antikörper gegen PD-1 und PD-L1 werden beim NSCLC in klinischen Studien untersucht.

Derzeit sind die beiden humanen IgG4-Antikörper gegen PD-1 (Nivolumab und Pembrolizumab) am weitesten klinisch entwickelt.

Nivolumab I In einer großen Phase-I-Dosisfindungs-Studie erhielten u.a. 129 NSCLC-Patienten nach multipler Vorbehandlung Nivolumab (1, 3 und $10 \mathrm{mg} / \mathrm{kg}$ ). Der Anti-PD-1-Antikörper wurde alle 2 Wochen verabreicht

- für maximal 96 Wochen oder

- bis zur Progression der Erkrankung oder

- bis zum Abbruch bei Unverträglichkeit.

In diesem prognostisch ungünstigen Kollektiv wurde eine objektive Ansprechrate von 17\% beobachtet. Das Toxizitätsprofil war akzeptabel [17]. Im 3-mg-Dosisarm (diese Dosis wird in weiteren Phase-II/III-Studien verwendet) lag das mediane Gesamtüberleben bei 14,9 Monaten, die Ein- und Zwei-Jahres-Überlebensrate bei $56 \%$ und $45 \%$.

Das Überleben war bei Patienten mit squamöser und nicht-squamöser Histologie ähnlich (Median 9,2 und 10,1 Monate). Darüber hinaus hing es nicht vom EGFR- oder KRAS-Mutationsstatus ab.

Nivolumab in der Erstlinientherapie | Eine weitere Phase-I-Studie schloss verschiedene Kohor-

\begin{tabular}{|c|c|c|}
\hline $\begin{array}{l}\text { Therapiegruppen- } \\
\text { vergleich }\end{array}$ & $\begin{array}{l}\text { medianes immun- } \\
\text { abhängiges progressions- } \\
\text { freies Überleben }\end{array}$ & $\begin{array}{l}\text { medianes } \\
\text { Gesamtüberleben }\end{array}$ \\
\hline $\begin{array}{l}\text { Ipilimumab-Chemo- } \\
\text { therapie simultan vs. } \\
\text { Chemotherapie }\end{array}$ & $\begin{array}{l}5,5 \text { vs. } 4,6 \\
\text { - HR } 0,81 \\
\text { - p-Wert } 0,13\end{array}$ & $\begin{array}{l}9,7 \text { vs. } 8,3 \\
\text { - HR } 0,99 \\
\text { - p-Wert } 0,48\end{array}$ \\
\hline $\begin{array}{l}\text { Ipilimumab-Chemo- } \\
\text { therapie phasisch vs. } \\
\text { Chemotherapie }\end{array}$ & $\begin{array}{l}5,7 \text { vs. } 4,6 \\
\text { - HR } 0,72 \\
\text { - p-Wert } 0,05\end{array}$ & $\begin{array}{l}12,2 \text { vs. } 8,3 \\
\text { - HR } 0,87 \\
\text { - p-Wert } 0,23\end{array}$ \\
\hline
\end{tabular}

HR: Hazard Ratio

Tab. 1 Ergebnisse der Phase-II-Studie [15].

ten Chemotherapie-naiver NSCLC-Patienten ein (CheckMate 012). Eine Interimauswertung zeigte bei Nivolumab-Monotherapie eine Ansprechrate von 30\% (alle Patienten) bei einem andauernden Ansprechen bei $67 \%$ dieser Patienten zum Zeitpunkt der Analyse [18].

Histochemie lässt auf Ansprechen schließen I In beiden klinischen Studien korrelierte eine starke immunhistochemische Färbung von membranösen PD-L1-Molekülen auf Tumorzellen mit einem verbesserten Ansprechen und längerem Gesamtüberleben. Allerdings wurde eine klinische Aktivität von Nivolumab auch bei PD-L1-negativen Tumoren beobachtet.

Nivolumab als späte Therapielinie | Eine aktuelle Phase-II-Studie an 117 Patienten mit Plattenepithelkarzinom zeigte für Nivolumab als Monotherapie in der 3. oder späteren Therapielinie (CheckMate 063) eine Ansprechrate von 15\% (95\%-KI $8,7-22,2)$. Das mediane Gesamtüberleben lag bei 8,2 Monaten (95\%-KI 6,05-10,91) [19].

Abb. 2 Checkpoint PD-1 (im Tumorgebiet). Über den T-Zell-Rezeptor erkennt die aktivierte T-Zelle das Tumorantigen. Die Zielzelle wird zerstört (Zytolyse). Die Tumorzelle exprimiert Liganden (PD-L1, PD-L2), die über den Rezeptor PD-1 die Immunantwort des TZR inhibieren. Antikörper gegen PD-1 und PD-L1 stellen die Immunantwort wieder her.

Abkürzungen: s. $\vee$ Abb. 1.

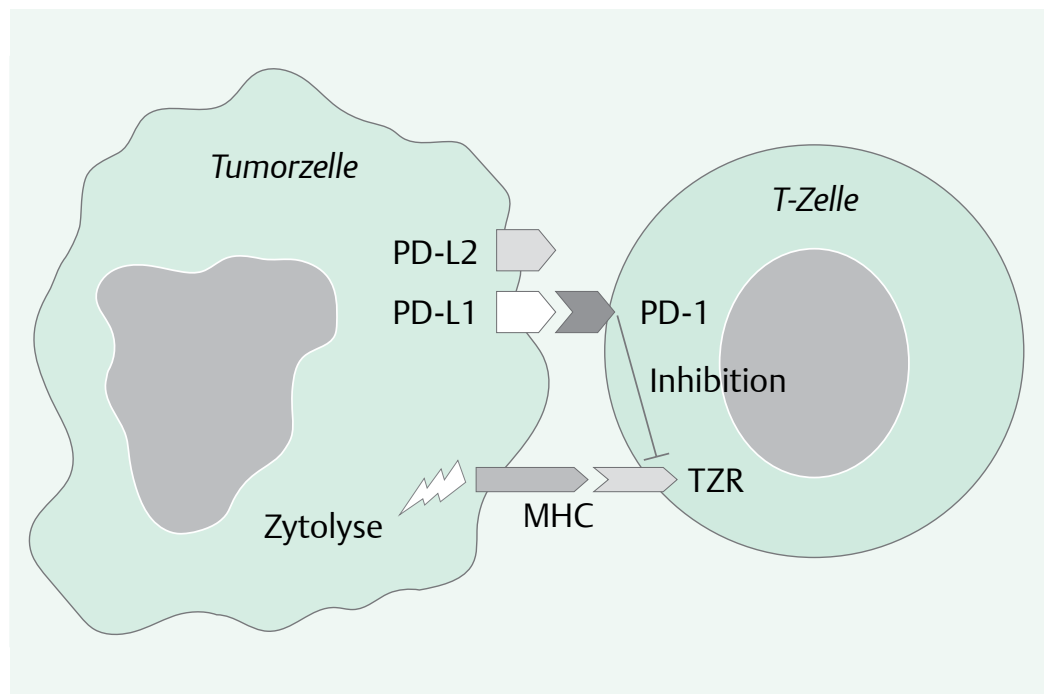


Aktuell untersuchen Phase-III-Studien bei Patienten mit PD-L1-positivem NSCLC

- die Wirkung einer Nivolumab-Monotherapie vs. einer Standardtherapie (Docetaxel) beim (nicht-)squamösen NSCLC

- eine Nivolumab-Monotherapie als Erstlinientherapie vs. Platin-haltige Kombinations-Chemotherapien (CheckMate 026).

Pembrolizumab bei vorbehandelten Patienten I Pembrolizumab ist ebenfalls ein humaner AntiPD1-Antikörper. Innerhalb einer großen Phase-IStudie (KEYNOTE-001) wurde ein Subkollektiv mit 250 vorbehandelten NSCLC-Patienten gebildet. Hier führte eine Pembrolizumab-Behandlung zu folgenden Ergebnissen:

- akzeptables Toxizitätsprofil

- Ansprechraten: 9-23\% - bei PD-L1-positiven und -negativen Tumoren [20].

- medianes Gesamtüberleben: 8,2 Monate (95\%-KI 7,3 Monate-nicht erreicht)

- medianes PFS: 10 Wochen (95\%-KI 9,1-15,3 Wochen).

- positives Anfärben membranöser PD-L1Moleküle korreliert mit Therapie-Ansprechen und Krankheitsverlauf

Pembrolizumab als Erstlinientherapie | Eine weitere Subgruppe in der KEYNOTE-001-Studie untersuchte Pembrolizumab als Erstlinientherapie bei PD-L1-positivem NSCLC: Bei 36\% der Patienten kam es zu einem Ansprechen nach „Immune related response“-Kriterien (s.u.) [21].

Pembrolizumab vs. Chemotherapie | Pembrolizumab wird aktuell in unterschiedlicher Dosierung vs. Docetaxel untersucht

- bei Krankheitsprogression nach Platin-haltiger Kombinations-Chemotherapie,

- in Kombination mit Cisplatin/ Pemetrexed oder Carboplatin/ Paclitaxel als Erstlinientherapie.

MPDL3280A | Der humane monoklonale IgG4Antikörper MPDL3280A richtet sich gegen PD-L1. In einer Phase-I-Expansionsstudie mit 85 NSCLCPatienten (squamöse und nicht-squamöse Histologie) wurde die Behandlung mit MPDL3280A in einer Dosierung von 1-20 mg/ kg untersucht. Die Studie kam zu folgenden Ergebnissen:

- Ansprechrate nach den Response Evaluation Criteria In Solid Tumors (RECIST): 23\%

- Krankheitsstabilität nach 6 Monaten bei $17 \%$ der Patienten

- PFS-Rate nach 6 Monaten: 46\% [22]

Die Expression von PD-L1 war prädiktiv für ein verbessertes Ansprechen bei allerdings sehr geringer Patientenzahl. Verschiedene Phase-II- und -IIIStudien schließen sowohl unselektionierte als auch selektiv PD-L1-positive NSCLC-Patienten ein.

MEDI-4736 | Ein anderer PD-L1-Antikörper, MEDI4736, wird in einer laufenden Phase-I-Dosis-Eskalationsstudie untersucht. Erste Daten zei- gen bei 3 von 13 vorbehandelten NSCLC-Patienten eine partielle Remission bei guter Verträglichkeit [23]. Verschiedene Expansionskohorten rekrutieren derzeit innerhalb dieser Studie Patienten mit soliden Tumoren einschließlich NSCLC.

BMS-936559 | Auch BMS-936559 ist ein AntiPD-L1-Antikörper, der bei NSCLC-Patienten untersucht wurde. Eine Phase-I-Studie schloss 75 Patienten mit vorbehandeltem NSCLC ein. Die Analyse der auswertbaren Daten $(n=49)$ ergab eine Ansprechrate von 12,5\%. Der Anteil der Patienten,die nach 6 Monaten noch progressionsfrei waren, betrug $31 \%$ [24].

BMS-936559 wird aufgrund dieser Ergebnisse derzeit nicht weiter entwickelt.

\section{Verträglichkeit}

Welche Nebenwirkungen sind zu erwarten? | Immunologisch vermittelte Komplikationen stehen im Vordergrund. Sie sind zumeist reversibel [15, $25,26]$. Insgesamt zeigten die bisher angesprochenen Substanzen ein akzeptables Toxizitätsprofil.

Nebenwirkungen von Ipilimumab | Im zeitlichen Verlauf führte Ipilimumab in Kombination mit Chemotherapie $\mathrm{zu}$

- Hautausschlag(10-25\%; persistiert3-4Wochen; bei $3 \%$ als schwerwiegend beschrieben [15])

- Diarrhoe $(23 \%)$

- Leberwert-Erhöhungen (40\%)

Weitere, seltenere Nebenwirkungen sind:

- Pneumonitis

- Hypophysitis

- Hypopituitarismus

- anaphylaktische Reaktionen

- Vitiligo

- Hepatitis

- Thyroiditis [25].

TSH, T3, fT4, Elektrolyte und Leberwerte sollten initial und vor jeder Dosis durchgeführt werden, um etwaige hormonale Defizite zu erfassen.

Nebenwirkungen von PD-1 und PD-L1 | Das Toxizitätsprofil von PD-(L)1-Inhibitoren ist ähnlich wie bei CTLA-4-Antikörper, es scheint in der Regel jedoch geringer zu sein. Relevante immunologisch vermittelte Nebenwirkungen im Gesamtkollektiv (mit anderen soliden Tumoren) waren

- Vitiligo (3\%)

- Pneumonitis (3\%)

- Colitis (11\%)

- Thyreoiditis (3\%)

- Fatigue $(24 \%)$

- Appetitlosigkeit (8\%)

- Anämie $(1 \%)$

- Nausea (8\%) [25] 


\section{Besonderheiten des Ansprechens unter Immuntherapie}

Radiologische Veränderungen | Eine Besonderheit bei den hier besprochenen Immuntherapien ist das Muster des Ansprechens. Verschiedene radiologische Veränderungen wurden in Zusammenhang mit einem günstigen Verlauf bei einer Immuntherapie gebracht:

- Schrumpfung der Indikatorläsion (klassisches Ansprechen)

- stabile Erkrankung mit einer nachfolgenden, langsamen Schrumpfung der Indikatorläsionen

- Ansprechen nach einer initialen Vergrößerung der Indikatorläsion

- Ansprechen der Indikatorläsion unter Auftreten neuer, infiltrativer Läsionen [27]

Die letzten beiden Varianten sind wahrscheinlich weniger durch eine initiale echte Tumorprogression, sondern eher durch entzündliche T-Zell-Infiltrate erklärt.

„Immune related response criteria“ I Resultierend aus diesen Erkenntnissen wurden sogenannte „Immune related response criteria“ entwickelt. Diese werten - im Gegensatz zu den WHO-Kriterien - die Entwicklung neuer Infiltrate nicht automatisch als Progression und fordern eine zweite Kontrolle nach vier Wochen, falls die Indikatorläsion zunimmt.

\section{Ausblick}

Die immunmodulierende Therapie mit Antikörpern scheint beim NSCLC ein vielversprechender Ansatz zu sein. Bei PD-1-Antikörpern steht mit der membranösen PD-L1-Expression womöglich auch ein prädiktiver immunhistochemischer Marker zur Verfügung. Zur abschließenden Beurteilung müssen hier allerdings die laufenden Phase-III-Studien abgewartet werden.
Unterschiedliche Testverfahren und Cut-Offs erschweren die Interpretation und Vergleiche verschiedener klinischer Studien. Die besondere Toxizität muss beachtet werden. Neben einer Unterbrechung der Therapie steht hier die antiinflammatorische Behandlung mit Steroiden im Vordergrund. Durch Kombinationen verschiedener Immuntherapeutika oder mit anti-angiogenen Substanzen oder Zytostatika könnte die Wirkung verstärkt werden. Diese Ansätze werden derzeit in frühen klinischen Studien evaluiert.

Die Immuntherapie könnte sich als neue Therapiemodalität mit noch nicht abschätzbarem Potenzial beim NSCLC etablieren. Die Ergebnisse der laufenden Phase-III-Studien sind allerdings abzuwarten.

Konsequenz für Klinik und Praxis

- Verschiedene immuntherapeutische Ansätze werden derzeit untersucht

- Antikörper gegen CTLA-4, PD-1 und PD-L1 sind am weitesten klinisch entwickelt

- Erste Daten zeigen für Monotherapie mit PD-1-Antikörpern bei unselektionierten, vorbehandelten NSCLC-Patienten Ansprechraten um 20\% und ein medianes Gesamtüberleben zwischen 8 und 15 Monaten.

- Die membranöse PD-L1-Expression auf Tumorzellen korreliert mit verbessertem Ansprechen und günstigem Verlauf

- Die Verträglichkeit ist allgemein gut D insbesondere immunologisch vermittelte Komplikationen müssen beobachtet und kontrolliert werden

- Ergebnisse von größeren klinischen Studien sind abzuwarten.

- Der Stellenwert des radiologisch kontrollierten Ansprechens ist zu entablieren.

\section{Literatur}

1 Bremnes RM, Al-Shibli K, Donnem T et al. The role of tumor-infiltrating immune cells and chronic inflammation at the tumor site on cancer development, progression, and prognosis: emphasis on non-small cell lung cancer. J Thorac Oncol 2010; 6: 824-833

2 Mantovani A, Allavena P, Sica A, Balkwill F. Cancerrelated inflammation. Nature 2008; 454: 436-444

3 Piersma SJ, Jordanova ES, van Poelgeest MI et al. High number of intraepithelial CD8+ tumor-infiltrating lymphocytes is associated with the absence of lymph node metastases in patients with large early-stage cervical cancer. Cancer Res 2007; 67: 354-361

4 Gooden MJ, de Bock $\mathrm{GH}$, Leffers $\mathrm{N}$ et al. The prognostic influence of tumour-infiltrating lymphocytes in cancer: a systematic review with meta-analysis. $\mathrm{Br}$ J Cancer 2011; 105: 93-103

5 De Pas T, Giovannini M, Rescigno $\mathrm{M}$ et al. Vaccines in non-small cell lung cancer: rationale, combination strategies and update on clinical trials. Crit Rev Oncol Hematol 2012; 83: 432-443

6 Shepherd FA, Douillard JY, Blumenschein GR Jr. Immunotherapy for non-small cell lung cancer: novel

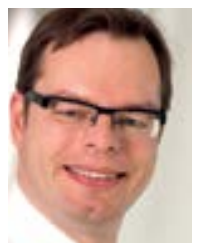

PD Dr. med. Niels Reinmuth ist Oberarzt in der Abteilung Onkologischer Schwerpunkt der LungenClinic Großhansdorf n.reinmuth@lungenclinic.de

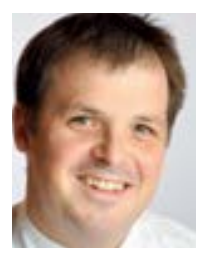

Dr. med. David F. Heigener arbeitet als Oberarzt in der Abteilung Onkologischer Schwerpunkt LungenClinic Großhansdorf d.heigener@lungenclinic.de

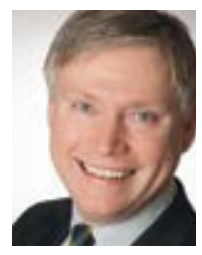

PD Dr. med. Martin Reck ist Chefarzt der Onkologie und Leiter des Lungenkrebszentrums LungenClinic Großhansdorf

m.reck@lungenclinic.de

approaches to improve patient outcome. J Thorac Oncol 2011; 6: 1763-1773

7 Bradbury PA, Shepherd FA. Immunotherapy for lung cancer. J Thorac Oncol 2008; 3 (6 Suppl. 2): S164-170

8 Cuppens K, Vansteenkiste J. Vaccination therapy for non-small-cell lung cancer. Curr Opin Oncol 2014; 26 : $165-170$

9 Butts CA, Socinski MA, Mitchell P et al. START: A phase III study of L-BLP25 cancer immunotherapy for unresectable stage III non-small cell lung cancer. J Clin Oncol 2103; 31: abstr 7500

10 Vansteenkiste JF, Cho B, Vanakesa T et al. MAGRIT, a double-blind, randomized, placebo-controlled Phase III study to assess the efficacy of the recMAGE-A3 + AS15 cancer immunotherapeutic as adjuvant therapy in patients with resected MAGE-A3-positive non-small cell lung cancer (NSCLC). Ann Oncol 2014; 25: abstract 11730

Vollständiges Literaturverzeichnis unter

http://dx.doi.org/10.1055/s-0041-100763
Interessenkonflikt

N. R.: Hoffmann-La Roche, Lilly, Novartis, Boehringer-Ingelheim, TEVA, Otsuka, MSD, BMS,

Amgen

D. H.: Hoffmann-La Roche, Lilly, Novartis, Boehringer-Ingelheim, Pfizer, BMS

M. R.: Hoffmann-La Roche, Lilly, Novartis, Boehringer-Ingelheim, AstraZeneca, MSD, BMS, Pfizer

DOI 10.1055/s-0041-100763

Dtsch Med Wochenschr 2015; 140: 329-333

(c) Georg Thieme Verlag KG . Stuttgart · New York . ISSN 0012-0472 
11 Giaccone G, Bazhenova L, Nemunaitis J et al. A phase III study of belagenpumatucel- $L$ therapeutic tumor cell vaccine for non-small cell lung cancer (NSCLC). European Cancer Congress 2013 2013: LBA 2

12 www.clinicaltrials.gov (letzter Zugriff: 8.11.2014)

13 Tsuji T, Altorki NK, Ritter $G$ et al. Characterization of preexisting MAGE-A3-specific CD4+ T cells in cancer patients and healthy individuals and their activation by protein vaccination. I Immunol 2009; 183: 4800-4808

14 Peggs KS, Quezada SA, Chambers CA et al. Blockade of CTLA-4 on both effector and regulatory T cell compartments contributes to the antitumor activity of anti-CTLA-4 antibodies. J Exp Med 2009; 206: 1717-1725

15 Lynch TJ, Bondarenko I, Luft A et al. Ipilimumab in combination with paclitaxel and carboplatin as first-line treatment in stage IIIB/IV non-small-cell lung cancer: results from a randomized, double-blind, multicenter phase II study. J Clin Oncol 2012; 30: 2046-2054

16 Velcheti V, Schalper KA, Carvajal DE et al. Programmed death ligand-1 expression in non-small cell lung cancer. Lab Invest 2013; 94: 107-116

17 Brahmer JR, Horn L, Gandhi L et al. Nivolumab (anti-PD-1, BMS-936558, ONO-4538) in patients (pts) with advanced non-small-cell lung cancer (NSCLC): Survival and clinical activity by subgroup analysis. J Clin Oncol 2014; 32: abstr 8112

18 Gettinger SN, Shepherd FA, Antonia SJ et al. First-line nivolumab (anti-PD-1; BMS-936558, ONO-4538) monotherapy in advanced NSCLC: Safety, efficacy, and correlation of outcomes with PD-L1 status. J Clin Oncol 2014; 32: abstr 8024

19 Ramalingam SS, Mazières ], Planchard D et al. Phase II Study of Nivolumab (anti-PD-1) in Patients with Advanced, Refractory Squamous Non-Small Cell Lung Cancer. 2014 Chicago Multidisciplinary Symposium on Thoracic Oncology 2014; abstr 3462
20 Garon EB, Gandhi L, Rizvi N et al. Antitumor activity of pembrolizumab (Pembro; MK-3475) and correlation with programmed death ligand 1 (PD-L1) expression in a pooled analysis of patients (pts) with advanced non-small cell lung carcinoma (NSCLC). Ann Oncol 2014; 25: LBA 43

21 Rizvi NA, Garon EB, Patnaik A et al. Safety and clinical activity of MK-3475 as initial therapy in patients with advanced non-small cell lung cancer (NSCLC). J Clin Oncol 2014;32: abstr 8007

22 Soria JC, Cruz C, Bahleda R et al. Clinical activity, safety and biomarkers of PD-L1 blockade in non-small cell lung cancer (NSCLC): Additional analyses from a clinical study of the engineered antibody MPDL3280A (anti-PDL1) European Cancer Congress 2013 2013: abstr 3408

23 Brahmer JR, Rizvi NA, Lutzky J et al. Clinical activity and biomarkers of MEDI4736, an anti-PD-L1 antibody, in patients with NSCLC. J Clin Oncol 2014; 32: abstr 8021

24 Brahmer JR, Tykodi SS, Chow LQ, et al. Safety and activity of anti-PD-L1 antibody in patients with advanced cancer. N Engl J Med 2012; 366: 2455-2465

25 Topalian SL, Hodi FS, Brahmer JR et al. Safety, activity, and immune correlates of anti-PD-1 antibody in cancer. N Engl J Med 2012; 366: 2443-2454

26 Heigener D, Reck M. Exploring the potential of immuno-oncology-based treatment for patients with non-small cell lung cancer. Expert Rev Anticancer Ther 2015; 15: 69-83

27 Wolchok JD, Hoos A, O‘Day S, et al. Guidelines for the evaluation of immune therapy activity in solid tumors: immune-related response criteria. Clin Cancer Res 2009; 15: 7412-7420 